Miklós Zrínyi : La Zrinyade ou Le Péril de Sziget, épopée baroque du XVII siècle

Villeneuve-d'Asq, Presses Universitaires du Septentrion, 2015. Introduction, traduction et notes de Jean-Louis VALLIN, postface de Farkas Gábor KISS.

Paul-Victor Desarbres

\title{
OpenEdition
}

Journals

Édition électronique

URL : https://journals.openedition.org/efo/5618

DOI : 10.4000/efo.5618

ISSN : 2275-1947

Éditeur

INALCO

Édition imprimée

ISBN : 978-2-343-08571-5

ISSN : 0071-2051

Référence électronique

Paul-Victor Desarbres, "Miklós Zrínyi : La Zrinyade ou Le Péril de Sziget, épopée baroque du xvıe siècle », Études finno-ougriennes [En ligne], 47 | 2015, mis en ligne le 07 juillet 2016, consulté le 20 septembre 2021. URL : http://journals.openedition.org/efo/5618 ; DOI : https://doi.org/10.4000/efo.5618

Ce document a été généré automatiquement le 20 septembre 2021

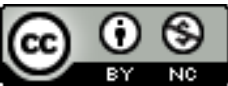

Études finno-ougriennes est mis à disposition selon les termes de la Licence Creative Commons Attribution - Pas d'Utilisation Commerciale 4.0 International. 


\section{Miklós Zrínyi : La Zrinyade ou Le Péril de Sziget, épopée baroque du XVII siècle}

Villeneuve-d'Asq, Presses Universitaires du Septentrion, 2015. Introduction, traduction et notes de Jean-Louis VALLIN, postface de Farkas Gábor KISS.

Paul-Victor Desarbres

1 L'histoire littéraire a décrété que «les Français n'ont pas la tête épique » (Gauthier 1841, p. 107); l'époque actuelle ne change rien à cet état des choses. JeanLouis Vallin a cependant eu le courage de traduire Le péril de Sziget de Miklós Zrínyi, longue épopée baroque en quinze chants d'une centaine de vers chacun. Il s'est vu décerner le prix Nicole Bagarry-Karátson pour la traduction des textes hongrois par un francophone. Les Presses Universitaires du Septentrion ont eu l'ambition de publier dans une utile et belle édition cette œuvre essentielle de la littérature hongroise, pétrie d'influences européennes classiques et modernes, et reflétant la question cruciale de l'affrontement avec les Turcs : à telle enseigne qu'on pourrait la dire centrale dans l'Europe du XVII siècle.

2 Traduire un texte déjà ancien de façon à le rendre accessible sans en trahir le sens, ni en ignorer la lettre : c'était la ligne de crête sur laquelle il a fallu se tenir. Le résultat aurait pu être rebutant, et ajouter aux difficultés que le texte présente pour le locuteur hongrois celles de la traduction. Il est particulièrement réussi, et inspire quelques réflexions sur ce type d'entreprise. Un double écart ou une double difficulté (l'altérité hongrois-français et l'archaïsme de la langue de Zrinyi) a amené Jean-Louis Vallin à prendre un parti susceptible de donner naissance non pas à une traduction trop contemporaine et destinée rapidement à l'obsolescence, ni à une traduction archaïsante mort-née ${ }^{1}$, mais à la tentative de reconstitution d'un équivalent pour la langue et la métrique: c'est ainsi le français «classique» de Corneille et Racine modernisé qui s'est imposé la plupart du temps (p. 20). On note aussi de menus archaïsmes dont il n'a cependant pas fait abus : « ne mettons à plus tard» (XIV, 93, v. 2) ou «t'éjouir grâce à moi » (XIV, 61, v. 2). À titre de licence poétique classique, on trouvera ainsi des «encor ${ }^{2} »$. Pour reprendre les mots d'un autre traducteur, il semble 
qu'il n'ait pas été question de "naturaliser", mais de "faire apparaître dans son altérité culturelle " l'œuvre (Ausone de Bordeaux, 2010, p. 24-34) : l'avant-propos et l'introduction de la Zrinyiade montrent un souci tout particulier de rendre accessible au public francophone les grandes caractéristiques de la langue hongroise ou de ses sonorités. Cette traduction est une vraie reconstitution, qui ne gomme pas l'altérité de la langue et de l'époque de son auteur. Dans cet ordre esprit, le traducteur déclare laisser tels quels tous les noms de villes ou de personnages, hongrois ou turcs, ainsi que les realia du monde turc qui n'ont pas d'équivalent en français - à l'exception bien compréhensible de Soliman qu'il ne signale pas : c'est en fait encore la règle d'Amyot d'après laquelle la notoriété entraîne une francisation du nom propre. Pour les noms livrés sans changement, cela permet d'ailleurs de profiter des variantes du hongrois dans la traduction, ainsi pour Sziget ou Szigetvár : ainsi « Szigetnek Hektora » devientil sans inconvénient « l'Hector de Szigetvár » (X, 94, v. 1).

\section{Traduire en vers}

Dans l'introduction, une courte présentation de Zrínyi, homme d'épée et de plume, précède un développement modestement intitulé «Point de vue du traducteur » qui nous fait entrer avec précision et sans fausses pudeurs dans la fabrique, la cuisine du traducteur (p. 17-24). Le choix du vers d'abord doit retenir l'attention (p. 17-18): on voudrait prolonger les justifications de Jean-Louis Vallin. Comme dans les anciennes chroniques hongroises, Zrínyi utilise des quatrains de dodécasyllabes monorimes. Le système de suffixation propre au hongrois permet au poète d'obtenir aisément des rimes. Le traducteur ne s'asservit pas à cette contrainte. Rejoignant les expérimentations de certains lettrés renaissants ${ }^{3}$, il propose un vers compté, mais non rimé. Il admet ainsi la césure épique, apocope systématique du e caduc en septième position du vers. C'est une réponse très satisfaisante au besoin de souplesse qu'éprouve tout traducteur - et tout lecteur - devant le mètre trop illustre. Cette simple modification permet d'éviter des contorsions et reste fidèle à la donnée fondamentale de l'alexandrin classique pratiqué jusqu'au $\mathrm{XX}^{\mathrm{e}}$ siècle : la bipartition du vers en deux hémistiches de six syllabes, une pause étant observée entre eux ${ }^{4}$. Cet invariant correspond à un besoin de reconnaissance du vers, que respecte parfaitement la traduction :

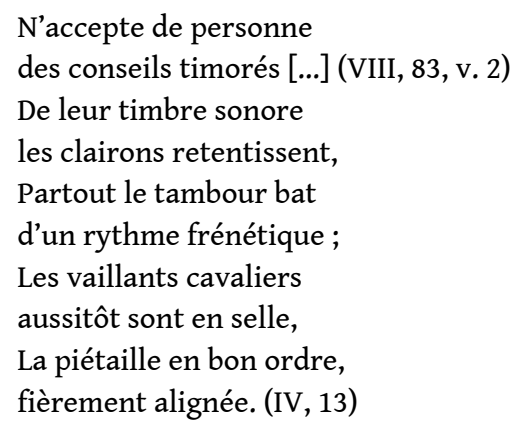

Le e caduc est ici parfaitement muet, sans que cela pose de difficultés de lecture, tant la double segmentation de l'alexandrin est un fait d'une régularité qui ne souffre pas d'exception. Combien de traducteurs pourraient s'inspirer de cette solution efficace et libératrice! Ce dernier passage mène à une seconde considération d'importance dans la traduction : à défaut de rime, le traducteur n'a pas négligé la prosodie des sonorités ${ }^{5}$. Le fait de renoncer à la rime et d'adopter une version souple de l'alexandrin classique 
offre une marge de liberté et la possibilité de construire des chaînes prosodiques comme dans l'exemple précédent (v. 2) où l'allitération espacée en [t] est suivie d'une allitération suivie en [b] provoquant deux accents prosodiques consécutifs : c'est le sémantisme du mot tambour qui est ainsi mis en valeur. Cette traduction porte aussi en elle d'autres allitérations qui l'apparentent à un français quasi renaissant ou prémalherbien, pour le plus grand bonheur du lecteur. Ainsi, la mésange «leurrée par le pipeau et vite prise au piège » (XII, 81, v. 2). Dans ces cas, le traducteur ne cherche pas à reproduire une allitération du texte hongrois, mais donne un discret relief propre à la couleur de son œuvre de traduction ${ }^{6}$.

5 Plus largement, le traducteur a pratiqué l'allitération ou l'assonance finale, ainsi qu'une forme de rime lâche qui convient parfaitement par rapport à l'original hongrois : les quatrains de Zrínyi sont rimés, mais la rime n'a pas le même poids dans le système d'accentuation du hongrois - où elle n'est presque jamais accentuée - qu'en français où elle l'est systématiquement. On trouve de temps à autre des rimes croisées :

Pál frappe Jahia Zaim,

qui laisse aller sa vie

Descendre sous la terre.

Mais à son tour, Beriel

De Pál tranche la gorge ;

implacable et cruel,

Son sabre goûte au sang

- et Pál y rend la vie. (XI, 87)

Régulièrement, deux vers d'un même quatrain, séparés ou côte à côte riment ensemble, comme dans cette description d'Alecto qui provoque le frisson:

Des yeux coule une écume sanglante et venimeuse,

Et son gosier exhale des vapeurs sulfureuses. (I, 29, v. 3-4)

Souvent, on lit quelques rimes que la nomenclature scolaire nommerait "pauvres ", éparses, et créant un discret et riche phénomène d'assonance ${ }^{7}$. De vagues homophonies («Demirham » / «Deliman", « répondre » / « nombre ») remplacent aussi parfois la rime.

Parfois c'est la répétition du même mot à la fin du vers qui fait office de rime ${ }^{8}$. Quand bien même ces répétitions se trouvent parfois dans l'original ${ }^{9}$, on peut se demander si ces "prosaïsmes» ne sont pas une façon ingénieuse de rendre, à une échelle bien moindre, l'effet de monotonie qui se dégage de ces poèmes hongrois à rime par suffixe. On conçoit tous les avantages et les libertés qu'offre un tel système. De quel droit ne profiterait-on pas de la rime entre "mer » et " père » (IX, 1) ? La rime, «ce bijou d'un sou ", n'a pas arrêté le traducteur, et c'est tant mieux.

Si l'entreprise n'était pas aussi réussie, on pourrait discuter sur un autre choix de traduction : pour certains syntagmes usuels du français, «les armes et le héros », on a jugé qu'on pouvait s'affranchir du décompte des syllabes en vigueur dans le reste du texte. On lirait ainsi : « les arm's et le héros ». Cela constitue dans la masse du texte des cas d'exception qui nuisent un peu à la régularité métrique et à l'effet de reconnaissance qu'elle induit. La césure épique était fructueuse parce que libératrice et aisément identifiable; on est plus réservé pour les autres cas où le e à la finale (apocope) ou à l'intérieur d'un mot (syncope) n'est pas compté10. Mais c'est un phénomène marginal. 


\section{Du vers à la recréation d'un style.}

10 On est introduit aux archaïsmes de la Zrinyiade avec pédagogie (p. 18-20) : à la parution de l'épopée dans un vaste recueil intitulé la Sirène de l'Adriatique en 1651 à Vienne, le hongrois n'a pas connu la grande révolution linguistique ou «nyelvújítás » du $\mathrm{XIX}^{\mathrm{e}}$ siècle, Zrínyi fait figure de défenseur et illustrateur de la langue hongroise (alors même qu'il aurait pu aussi écrire en croate); il se plaint, comme tant d'auteurs et traducteurs français de la Renaissance, de la pauvreté de son vulgaire ("Au lecteur », p.41). Le dernier sous-développement de l'introduction, modestement titré «Ressources de la traduction » fait entrer dans le vif des choix de traduction (p. 20-24). Différentes recettes sont exposées, qui peuvent s'avérer utiles pour les traducteurs amateurs. Il s'agissait tantôt de «compenser la longueur d'un mot hongrois »: ici, le traducteur retrouve les fameux binômes dont on avait pu abuser dans les traductions de la seconde moitié $\mathrm{du} \mathrm{XvI}{ }^{\mathrm{e}}$ siècle ${ }^{11}$ (Buridant 2002). Le principe de commutabilité des catégories grammaticales est aussi exposé. Deux tendances contradictoires, celle de la parataxe et celle la subordination, ne pouvaient être reproduites dans la traduction. On est cependant frappé par le soin (qu'illustre d'ailleurs un propos sur l'emphase) de restituer dans la mesure du possible un rythme de présentation des mots du texte hongrois. Le poète décrit ainsi Cumilla, amante du preux Delimán :

Nyughatatlankodik, vagy jár, vagy áll, vagy ül [...]

Marchant, debout, assise, le repos l'abandonne. (XII, 23, v. 3)

11 La simple inversion de la séquence ternaire et de l'hémistiche et la transformation des trois verbes en trois formes susceptibles d'être apposées au pronom personnel illustre cette technique de traduction. On ne perd ainsi rien des mouvements de la phrase ou des comparaisons épiques qui font la saveur de ce texte.

Le traducteur déclare aussi très simplement ne pas avoir hésité à utiliser certaines chevilles: il est possible que certains passages de l'original n'en aient pas été dépourvus. L'idée d'une poésie quintessentielle, à la Baudelaire ou plus encore à la Valéry, est bien étrangère à cette époque. Cela justifie quelques ajouts :

Fáti, édes anyám, elvesztem elmémet.

Fáti, ma douce mère, vois, j'en perds la raison [...] (XII, 29, v. 3)

13 Les techniques permettant de construire ces vers réguliers rapprochent ainsi le traducteur de modèles de la littérature classique française dont on sent qu'il a été imprégné. Jean-Louis Vallin a su recréer un style de Zrinyi en français, une voix visant l'efficacité épique, «sans façon »:

Que cette forteresse, ici, soit notre gloire

Ou n'ayons pour cercueils que des ventres d'oiseaux;

Mais du moins, soyons hommes et soyons des héros ;

Qu'ainsi notre beau nom dure éternellement. $(\mathrm{V}, 34)$

14 La redondance des compléments de lieu du texte initial est ici respectée. Ce qu'on a analysé comme le « sublime cornélien » n'est pas loin sur le fond.

15 On voudrait noter la formule de conclusion du chant IX, après la bataille, où le poète fait revivre l'exotisme et le fantasme magiques, avant d'abréger sur un prosaïsme plein de superbe, digne de la Légende des Siècles :

$\mathrm{Au}$-dessus des tombeaux, les hadjis, par leurs cris,

Encourageaient chacun à ne pas perdre espoir ;

Derviches, talismans évoquaient Mahomet 
Pour qu'il leur vînt en aide

- puis on les laissa là. (IX, 101)

En respectant globalement l'ordre des mots hongrois, le traducteur a su faire œuvre, et trouver des formules équivalentes dans la culture littéraire francophone. Il semble que ce soit d'ailleurs l'intuition au cœur de la création du prix Nicole Bagarry-Karátson que de récompenser l'utilisation des qualités de maîtrise de la langue et de la littérature françaises au profit de la transmission des textes hongrois.

\section{Un instrument d'étude}

Un résumé reprend l'action de chaque chant avec précision (p. 25-35); un dossier d'illustrations qui donnent un aperçu de la diffusion et de la postérité de l'histoire et du mythe de Zrinyi ainsi que de l'œuvre de son petit-fils (p. 110-114 ; p. 184-188) ; un index des personnages historiques mentionnés sera utile aux chercheurs intéressés par les turcica de l'âge baroque : on y trouve la forme courante de certains noms en français (p. 285-286) ; deux cartes des lieux mentionnées rendent la géographie du bassin des Carpathes accessible au lecteur. Les notes sur le texte, en nombre volontairement réduit, sont éclairantes : aurait-on voulu qu'il soit davantage fait état des modèles gréco-latins ou italiens de Miklós Zrínyi ? Une postface de Farkas Gábor Kiss resitue l'œuvre dans la littérature hongroise (p.275-284): première épopée d'inspiration classique dans cette langue, la Zrinyiade est tributaire de chroniques rimées, de la littérature croate anti-turque, et bien sûr de modèles italiens, grâce aux zones d'influence du nord de l'Adriatique : l'Arioste, le Tasse et le cavalier Marino ne sont pas loin. Deux textes de ce dernier semblent même avoir été déterminants pour le poète qui réussit à transformer une défaite en épopée ${ }^{12}$. Une bibliographie complète donne accès aux principales éditions, traductions du texte et études. On perçoit ce qu'il y a d'expérience de l'enseignement dans la présentation même de ce livre. Les comparatistes n'ont plus qu'à se mettre au travail.

\section{BIBLIOGRAPHIE}

AUSONE DE BORDEAUX 2010, « Notes sur la traduction », CEuvres complètes, Bordeaux : éd. Bernard Combeaud, p. 24-34.

BURIDANT Claude 2002, « Les paramètres de la traduction chez Blaise de Vigenère », in Blaise de Vigenère, poète et mythographe au temps de Henri III, dir. CAZAURAN Nicole, Paris Presses de l'École normale supérieure (Cahiers Verdun-Louis Saulnier).

CORNULIER Benoît de, 1982, Théorie du vers. Rimbaud, Verlaine, Mallarmé, Paris : Éditions du Seuil. DESSONS Gérard, MESCHONNIC Henri, 1998, Traité du rythme. Des vers et des proses, Paris : Dunod. GAUTHIER Théophile, 1841, «La Divine Épopée, de M. Soumet », in La Revue des deux mondes, $4^{\mathrm{e}}$ série, tome 26 , p. 107-126. 


\section{NOTES}

1. Dans le souci de proposer une traduction rendant les archaïsmes épiques du Tasse, on a ainsi pu donner jour à une traduction difficile d'accès: TASSO Torquato, $L a$ Jérusalem libérée, éd. et trad. Orcel Michel, Paris, Gallimard, 2002.

2. Dans un seul cas (XV, 91, v. 2) l'orthographe « encor » semble cependant inutile, vu l'adoption de la « césure épique » (cf. infra).

3. Vigenère Blaise de, Le Psautier de David (1588), éd. Blum-Cuny Pascale, Paris, Le Miroir volant, 1991-1996, t. I, p. IX et 18.

4. Les trimètres romantiques ou les "tigresse[s] épou-vantable[s] d'Hycarnie " décadentistes sont justement des cas de fructueuse mise en conflit de la syntaxe et du mètre composé de deux hexasyllabe; il faut que la structure métrique soit maintenue en même temps que la structure syntaxique pour qu'il ait un effet, et non plate réduction aux seules exigences de la syntaxe et donc à la prose. Sur le vers constitué d'une égalité de segments syllabiques, cf. Cornulier 1982; pour une théorie plus globale : Dessons, Meschonnic 1998.

5. On utilise ce terme dans le sens de Dessons, Meschonnic, 1998, p. 167-172: pour l'essentiel, il s'agit des allitérations fondées sur la consonne ouvrante d'une syllabe, formant une chaîne sonore, à l'échelle du vers, du poème et de l'œuvre.

6. Voir aussi XI, 88, v. 2 ; XI, 96, v. 4 ; XI, 100, v. 4.

7. Voir I, 98 ; IV, 59 ; IV, 93 ; VIII, 88 ; XI, 2 et 3 ; XI, 88 ; XIII, 96 ; XIV, 20.

8. Voir XV, 90 et 93 ; IV, 112.

9. Le texte de la Zrinyiade n'est cependant pas exempt de répétitions: IX, 6 (nom propre répété aussi dans la traduction), VIII, 98 (« vitéz » qui devient « vaillant » puis «preux »), etc.

10. I, 2, v. 1 ; VIII, 92, v. 2 ; IX, 66, v. 4 ; X, 105, v. 2 ; XII, 108, v. 4 ; XII, 110, v. 2 ; XIII, 6, v. 2 (ne pourrait-on écrire « entre » au lieu de «d'entre»?); XIV, 61, v. 2 ; en XIV, 83, v. 3, il manque sans doute une syllabe?

11. Il s'agit d'une tendance accentuée chez beaucoup de traducteurs de la fin du $\mathrm{XVI}^{\mathrm{e}}$ siècle.

12. On peut objecter à l'auteur de l'essai que la chanson de geste n'est pas incompatible avec la défaite mais implique au moins le châtiment des méchants, ce qui est le cas de Soliman (et du Ganelon de la Chanson de Roland), mais cela ne remet pas en cause cette stimulante analyse sur la spécificité de cette épopée et son rapport à différents modèles. 
INDEX

Index géographique : Adriatique (mer), Szigetvár

motsclesru ЭПОС, БАРОККО, ПЕРЕВОД, ВЕРСИФИКАЦИЯ

Keywords : Epic, Baroque, Translation, Versification

nomsmotscles Français, Hongrois, Turcs

motscleset eepos, barokk, tõlge, värssimõõt

Thèmes : littérature, littérature hongroise

Index chronologique : XVIIe siècle, XXIe siècle

Mots-clés : épopée, baroque, traduction, versification 